\title{
Computational Aerothermodynamic Assessment of Space Shuttle Orbiter Tile Damage - Open Cavities
}

\author{
Maria V. Pulsonetti ${ }^{*}$ and William Wood ${ }^{\dagger}$ \\ NASA Langley Research Center, Hampton, VA, 23681
}

\begin{abstract}
Computational aerothermodynamic simulations of Orbiter windside tile damage in flight were performed in support of the Space Shuttle Return-to-Flight effort. The simulations were performed for both hypervelocity flight and low-enthalpy wind tunnel conditions and contributed to the Return-to-Flight program by providing information to support a variety of damage scenario analyses. Computations at flight conditions were performed at or very near the peak heating trajectory point for multiple damage scenarios involving damaged windside acreage reaction cured glass (RCG) coated silica tile(s). The cavities formed by the missing tile examined in this study were relatively short leading to flow features which indicated open cavity behavior. Results of the computations indicated elevated heating bump factor levels predicted for flight over the predictions for wind tunnel conditions. The peak heating bump factors, defined as the local heating to a reference value upstream of the cavity, on the cavity floor for flight simulation were $67 \%$ larger than the peak wind tunnel simulation value. On the downstream face of the cavity the flight simulation values were $60 \%$ larger than the wind tunnel simulation values. On the outer mold line (OML) downstream of the cavity, the flight values are about $20 \%$ larger than the wind tunnel simulation values. The higher heating bump factors observed in the flight simulations were due to the larger driving potential in terms of energy entering the cavity for the flight simulations. This is evidenced by the larger rate of increase in the total enthalpy through the boundary layer prior to the cavity for the flight simulation.
\end{abstract}

\section{Nomenclature}

$\begin{array}{llll}\text { BF } & \text { heating bump factor } & \text { d } & \begin{array}{l}\text { diffusion } \\ \text { edge }\end{array} \\ \text { CL } & \text { centerline } & \text { e } & \text { wall } \\ \text { Cp } & \text { coefficient of pressure } & & \\ \text { H } & \text { height of cavity, total enthalpy } & \\ \text { L } & \text { length of cavity, length of vehicle } & \\ \text { M } & \text { Mach number } & \\ \text { PH } & \text { peak heating } & \\ \text { q } & \text { heat transfer rate } & \\ \mathrm{Re}_{\theta} & \text { nomentum thickness Reynolds number } \\ \text { W } & \text { width } & \\ \mathrm{X} & \text { streamwise coordinate } & \\ \mathrm{Y} & \text { spanwise coordinate } & \\ \delta & \text { boundary layer thickness } & \end{array}$

\footnotetext{
* Aerospace Engineer, Aerothermodynamics Branch, Mail Stop 408A.

${ }^{\dagger}$ Aerospace Engineer, Aerothermodynamics Branch, Mail Stop 408A, Member AIAA.
} 


\section{Introduction}

Immediately following the loss of the Space Shuttle Columbia on February 1, 2003 there were a number of computational aeroheating ${ }^{1,2,3}$ efforts to aid in the understanding of what had occurred. These studies were focused on damage to the RCC leading edge panels on the left wing. The studies showed the effects on the flowfield of missing a full or partial RCC panel. The results agreed with the experimental data, providing further evidence that damage leading to a missing or partially missing RCC panel in the vicinity of panel 8 or 9 led to the loss of the vehicle.

In the Space Shuttle Return-to-Flight effort, which began after the release of the Columbia Accident Investigation Report ${ }^{4}$ in August of 2003, much of the focus was on the aerothermodynamic effects of damaged or missing Orbiter windside acreage tile. One of the aspects of the Return-to-Flight effort focused on improving the accuracy of cavity heat prediction technique. A series of wind tunnel tests in a low enthalpy facility to understand the aeroheating issues of wind tunnel test geometries more representative of missing tile or tile arrays were performed. A high fidelity viscous computational fluid dynamic (CFD) technique were used to support the effort to predict aeroheating of damaged tile in flight.

The wind tunnel tests ${ }^{5}$ were performed in the Mach 6 Air facility at NASA Langley. These experiments could match the momentum thickness Reynolds number $\left(\mathrm{Re}_{\theta}\right)$ and edge Mach numbers for a variety of different points on the windside of the vehicle at conditions near the peak heating point on the re-entry trajectory. The wind tunnel experiments, although helpful in their initial screening of the cavities, could not address the effects of high enthalpies flows over the cavities, as would be observed in flight. It was determined that high fidelity CFD would be used to help support the effort of extrapolating of the data to flight. The goal of the computational effort was to first validate the CFD at the wind tunnel conditions and then using the same methodology for running the cases, perform the flight calculations at the high enthalpy conditions, taking into account the real gas effects. This paper examines the traceability of the wind tunnel simulations to flight. It will present the patterns and magnitude of heating and the flow phenomena associated with open cavities at wind tunnel and flight conditions. It will also examine the differences in the heating pattern and magnitude between wind tunnel and flight and the causes of these differences.

\section{Computational Code}

The computations were performed using the Langley Aerothermodynamic Upwind Relaxation Algorithm (LAURA) code. ${ }^{6,7}$ LAURA is a CFD tool specialized for hypersonic re-entry physics and chemistry. LAURA has been validated against Orbiter laminar aerothermodynamic flight data ${ }^{8}$, and re-entry cavity studies on a cpasule ${ }^{9}$ It is a three-dimensional upwind finite volume solver with perfect gas, equilibrium and finite rate chemistry models. The code can solve the inviscid, thin-layer Navier-Stokes or full Navier-Stokes governing equations. Pointrelaxation is used to integrate in time to steady state. Roe-averaging ${ }^{10}$ with Harten's entropy fix ${ }^{11}$ and Yee's Symmetric Total Variation Diminishing (TVD) limiter ${ }^{12}$ is used to solve the inviscid fluxes while a second order scheme solves the viscous fluxes.

The wind tunnel computations were performed assuming a perfect gas due to the low temperatures of the flowfield. The wall was assumed to be at a constant temperature of $300 \mathrm{~K}$. The experimental data are presented in terms of the non-dimensional ratio of heat transfer coefficient to Fay-Riddell ${ }^{13}$ heat transfer coefficient at $300 \mathrm{~K}$. This ratio is assumed to remain constant with wall temperature. Laminar calculations were performed using both full Navier-Stokes as well as a thin-layer formulation of the Navier-Stokes equations.

The flight computations assumed the flowfield to be in chemical nonequilibrium (5 reacting species) and thermal equilibrium. Laminar calculations were performed using both full Navier-Stokes as well as a thin layer formulation of the Navier-Stokes equations. All solid surfaces were treated as partially catalytic to recombination using the reaction rates for reaction cured glass (RCG) from Stewart ${ }^{14}$. The calculations were preformed for a radiative equilibrium wall temperature, with an assumed surface emissivity of 0.89 .

\section{Open Cavities}

This paper will focus on shorter cavities where open cavity behavior was observed. For these cavities, the ratio of the cavity depth to the boundary layer thickness approaching the cavity was greater than 0.3 and the ratio of the cavity length to the boundary layer thickness approaching the cavity was less than 7.5. Open cavities are defined $^{15.16}$, as cavities that are sufficiently short such that the flow approaching the cavity begins to expand downward toward the cavity floor, but with the limited length of the cavity, strikes the downstream wall of the 
cavity. Part of this flow is then turned downward to reattach on the cavity floor. A recirculation bubble is created that occupies most of the cavity. Figure 1 shows a schematic of a typical open cavity two-dimensionally.

\section{Wind Tunnel Simulations}

Wind tunnel run 247 was chosen for this study as it had the highest signal to noise ratio of all the open cavity runs. The wind tunnel simulations were performed on grids which had a Cartesian-aligned H-grid within the cavity. These simulations were used to validate the CFD tools and the methodology with the experimental results. The computations were performed using a full Navier-Stokes formulation of the governing equations. The Cartesianaligned cavity grid for wind tunnel run 247 is shown in figure 2 and a magnified view of the grid highlighting the grid inside the cavity is shown in figure 3 . The grid had clustered cells on all viscous surfaces as well as in the vicinity of the shear layers just above the cavity. The grid had 3 million cells with 281,000 inside the cavity.

For the flight simulations of damage to the Orbiter windside, a Cartesian-aligned H-grid topology was not practical due to the variations of local OML grid topology at various points on the surface. Instead, a grid morphing tool $^{17}$ was developed whereby the local volume grid in a region was removed to be replaced with an O-grid whose surface matched the damaged cavity shape. To assure that this method for flight would produce correct results, the morphed grid topology was used for some of the wind tunnel simulations and the results compared to the H-grid computations. Additionally, as the full Navier-Stokes simulations are time intensive, it was determined to attempt to use a thin-layer formulations of the Navier-Stokes equations for the morphed grid at tunnel conditions to see the effect of the modeling of the governing equations. The morphed grid used to simulate wind tunnel run 247 is shown in figure 4 and a magnified view of the grid highlighting the grid inside the cavity is shown in figure 5 . As in the case of the Cartesian-aligned grid, the grid had cell clustered normal to all viscous surfaces as well as in the vicinity of the shear layers just above the cavity. The morphed grid for run 247 had 1.7 million cells with 424,000 cells inside the cavity. For all the Cartesian-aligned and morphed grid results presented in this paper, the stretching was limited to 1.15 and the cell Reynolds number at the wall had an approximate value of 1. Experience has shown that with this code and for these types of conditions, those values produce accurate heat transfer rates. Iterative convergence of the solutions was determined by examining the variations in the local heating over time. Convergence was assumed when the heating varied by less than $1 \%$ over 1000 iterations. For this wind tunnel simulation, the heating varied by less that $0.8 \%$ over 1000 iterations. Figure 6 shows the results from the Cartesianaligned $\mathrm{H}$-grid while figure 7 shows the results of the morphed O-grid at tunnel conditions, referred to as the baseline wind tunnel simulation. The edge properties are shown in table 1 . The freestream tunnel values of Reynolds number, Mach number and angle of attack were 1.1 million/ft, 5.9 and -10 degrees, respectively. The cavity dimensions are given in table 2 . In these images, as in all the contour images in this paper, the flow direction is from the lower left to the upper right corner of the figure. The cavities are shown in this orientation so that a cavity sidewall and the vertical downstream face of the cavity are visible. The contours show the heating bump factor defined as the local heating normalized by the heating value just upstream of the cavity. Nearly identical results were observed for the two simulations establishing grid topological independence of the solution.

Higher heating bump factors are observed on the upper portions of the downstream face of the cavity and on the OML just downstream of the cavity. These regions nears the upper downstream corner are referred to as the downstream lip. For these simulations, as with all the wind tunnel and flight simulations presented in this paper, all the cavity corners are modeled by 90 degree angles. Damage would not likely produce a cavity with perfect 90 angles. If any such angles initially existed they would be quickly smoothed by the high heating that would exist at the sharp corners. The CFD was modeled that way to ease of grid creation as this method is to aid in real time assessment of damage in flight. All heating values discussed are taken from points about $10-20 \%$ removed from the actual corners. When the downstream lip of the cavity is referred to in this paper, it corresponds to locations near the downstream lip of the cavity excluding the very near region to the actual corner.

Grid convergence was also investigated. A finer grid of 3.2 million cells with 920,000 cells inside the cavity was also run to assess the grid convergence. The heating bump factor contour plot of the fine grid result is shown in figure 8. The contours appear identical in heating patterns and magnitude to the results of the baseline simulation shown in figure 7. Figure 9 shows cuts through the centerline and peak heating portions of the cavity in the streamwise direction for the baseline and fine grid solution. The cuts are at about the same spanwise station for the two simulations. The results overplot indicating grid convergence.

A comparison was also made on the morphed wind tunnel simulations to assess the effect of the assumption of a thin-layer formulation of the governing equations. Figure 10 shows the heating bump factor contours of the simulations performed on the baseline morphed grid for the full Navier-Stokes formulation of the governing 
equations. The contours indicate the heating pattern and level was consistent with the baseline thin-layer NavierStokes computations shown in figure 7. Figure 11 shows cuts through the centerline and peak heating portions of the cavity in the streamwise direction for both the thin-layer and the full Navier-Stokes simulations at approximately the same spanwise locations. The cuts look almost identical.

\section{Flight Simulations}

With the morphed grid approach having been verified for the wind tunnel simulations, flight computations using the morphed grid approach were initiated. The flight simulations were performed at various CFD trajectory points and at various locations on the Orbiter windside. Figure 12 shows the locations where flight simulations were run. The flight simulations were performed for cavities scaled from the wind tunnel run 247. The scaling up of the cavity dimensions to the flight conditions was performed by increasing the wind tunnel cavity dimensions by the ratio of the boundary layer thickness of that location in flight to the wind tunnel value. This scaling was suggested in historical studies ${ }^{15,16,18-22}$. The boundary layer edge was assumed at $99.5 \%$ of the freestream total enthalpy. The scaling was performed on the length, width and depth of the cavities. An attempt was made to run the flight simulations at locations with edge Mach numbers and momentum thickness Reynolds numbers which matched the wind tunnel values. This was achieved for the off centerline Orbiter windside cavity simulation. For the Orbiter windside centerline cavity simulations, it was not always possible to exactly match the edge conditions due to the thickness of the tile and the transition criteria. For the centerline cases, if both conditions were not able to be matched exactly, more emphasis was placed on matching the edge Mach number as this was shown to be the more sensitive parameter for scaling the heating bump factors.

\section{Baseline Flight Simulation}

Figure 13 shows the morphed grid which was generated using MORPH for the run 247 flight simulations at the centerline location of non-dimensional axial coordinate, $\mathrm{X} / \mathrm{L}$, of 0.15 . This corresponds to a point on the windside centerline 190 inches downstream of the vehicle nose. A magnified view of the cavity grid is shown in figure 14 . From this figure, the clustering of the cells off the viscous surfaces and shear layers can be seen. The cavity was placed on the windside centerline of the vehicle to minimize the complication of streamwise curvature and spanwise pressure gradient effects. The cavity was aligned with the streamwise coordinate of the vehicle. The full cavity was run, rather than a half cavity with a symmetry plane down the middle of the cavity, in order to allow the simulation to pick up any asymmetric behavior. The cavity simulation was run at CFD point 1, a trajectory point on the Orbiter STS-107 re-entry trajectory, given in table 3. This simulation is referred to as the baseline flight simulation. The CFD point 1 flight condition corresponds to 404 seconds from entry interface (EI). This cavity, like all flight cavities, had dimensions scaled from the wind tunnel dimensions using the ratio of the local flight boundary layer thickness to the wind tunnel boundary layer thickness. For this CFD trajectory point and at this location on the vehicle, the edge properties and cavity dimensions are given in tables 1 and 2, respectively. As for the wind tunnel simulations, convergence was assumed when the heating varied by less than $1 \%$ over 1000 iterations. For this simulation, the variation over 1000 iterations was less than $0.8 \%$. This was typical of the level of iterative convergence achieved. Figure 15 shows the heating bump factors contours, plotted at the same levels as the wind tunnel simulations. Similar to the wind tunnel simulations, increased heating over the reference value is observed at the downstream portion of the cavity floor and at the upper portion of the downstream face of the cavity, spilling onto the downstream OML.

Grid convergence was also investigated. The baseline computation, mentioned above, was performed on a grid with 1.7 million cells with 583,000 cells inside the cavity. A finer grid of 3.8 million cells with 1.3 million cells inside the cavity was also run to assess the grid convergence. The heating bump factor contour plot of the fine grid is shown in figure 16. The contours appear identical in heating pattern and magnitude to the baseline simulations of figure 15. Figure 17 shows cuts through the centerline and peak heating portions of the cavity in the streamwise direction of the baseline and fine grid simulations, at approximately the same spanwise locations. The cuts show only a small variation in the heating bump factor levels, with an average percent difference of 2.9 percent.

As the flight simulations required more run time to establish convergence than the wind tunnel computations, there was a desire to run the simulations using a thin-layer formulations of the governing equations. From previous work it was observed that the thin-layer computations required significantly less run time to converge than the full Navier-Stokes computations. A comparison was therefore made early in the study to assess the effect of utilizing the thin-layer formulation of the governing equations for the flight simulations. Figure 18 shows the heating bump factor contours of the simulation performed on the baseline morphed grid for the full Navier-Stokes formulation of 
the governing equations. Comparing these results to the thin-layer Navier-Stokes simulation, figure 15, indicate that the heating pattern and level was consistent for the two simulations. Figure 19 shows cuts through the centerline and peak heating portions of the cavity in the streamwise direction for the two simulations at approximately the same spanwise stations. The cuts show only a small variation in the heating bump factor levels. From this comparison and other comparisons of thin-layer and full Navier-Stokes simulations, it was determined that a thinlayer formulation of the governing equations was sufficient for the purposes of this Return-to-Flight study.

The cause for the increased heating in the cavity over the reference value just upstream of the cavity can be shown in figure 20 which shows the streamlines about the cavity. The heating on the downstream face and lip of the cavity is due to the flow impingement as the flow passing over the cavity is turned toward the cavity. The flow striking the downstream face is then redirected downward to strike and reattach on the cavity floor, as observed in the heating footprint on the downstream portion of the cavity floor. The flow then proceeds upstream and as it approaches the upstream face of the cavity lifts off the cavity floor to be redirected upward. The flow is then turned downstream by the momentum of the flow passing over the cavity. This circular movement of the flow inside the cavity is typical of open cavity behavior.

\section{Differences in BF's between Wind Tunnel and Flight}

While the wind tunnel and flight simulations show similar behaviors in terms of the heating bump factor patterns, the magnitude between the two simulations are significantly different. Figure 21 shows heating bump factor distributions for both the wind tunnel and flight simulations. These distributions were taken through the streamwise centerline of the cavity and through the peak heating portion of the cavity. The heating bump factors in the flight cavity are significantly higher than the wind tunnel values inside and downstream of the cavity. The most important values, however, are the percent increases in heating bump factors in the higher heating regions where the material may or may not reach a critical heating limit. The peak bump factors on the cavity floor for flight simulation were $67 \%$ larger than the peak wind tunnel simulation value. On the downstream face of the cavity the flight simulation values were $60 \%$ larger than the wind tunnel simulation values. On the OML downstream of the cavity, the flight values are about $20 \%$ larger than the wind tunnel values.

\section{Flight Simulation at CFD pt. 2}

Another computation was performed for a scaled cavity at the same location on the Orbiter windside for CFD point 2 on the STS-107 re-entry trajectory, given in table 3. The edge properties and cavity dimensions are given in tables 1 and 2, respectively. A heating bump factor contour of this simulation is shown in figure 22. You can see that the heating bump factor and the heating patterns are very similar to the simulation at CFD point 1, figure 15 . This can also be seen in figure 23 which shows the centerline and peak heating axial cuts through the cavities for the wind tunnel and the two flight simulations. The magnitudes of the heating bump factors are very similar for the majority of the cavity, even though they were run at different trajectory points.

\section{Effect of Pressure Gradient}

An additional flight cavity simulation was performed on the Orbiter windside centerline at CFD point 1 on the STS-107 trajectory at a non-dimensional (X/L) axial location of 0.05, corresponding to a location 63 inches from the vehicle nose. This computation was performed to assess the effect of the streamwise pressure gradient on the heating bump factor. The edge properties and cavity dimensions are given in tables 1 and 2, respectively. The streamwise pressure gradient $(\mathrm{dCp} / \mathrm{dx}$ ) for this cavity location was $-0.2221 / \mathrm{m}$. However, the value of more importance is the scaled pressure gradient which is the product of the boundary layer thickness and the streamwise pressure gradient. This value is the gradient over the length of the cavity. For this simulation, the scaled pressure gradient was -0.0081 . In comparison, for the baseline flight simulation, at CFD point 1 and $X / L=0.15$, the streamwise pressure gradient was $-0.0671 / \mathrm{m}$ and the scaled streamwise pressure gradient was -0.0040 . Therefore, there is a factor of 2 difference in the scaled streamwise pressure gradients between the two cases. The results however, are nearly identical. Figure 24 shows the heating bump factor contours for the simulations at $\mathrm{X} / \mathrm{L}=0.05$. Comparison with figure 15, which shows the heating bump factors for the baseline computation, indicates that the heating bump factor patterns and magnitudes nearly identical. This can also be observed from figure 25 which is identical to figure 23 except that it adds the cuts from this simulation. The cuts from the flight simulations at CFD point 1 at $\mathrm{X} / \mathrm{L}=0.05$ and 0.15 are very similar for the majority of the cavity, with a percent difference of about $6 \%$, except for a small portion of the downstream region of the cavity floor for the centerline cuts where the percent difference reaches $18 \%$. For most of the regions of the cavity, however, the heating bump factors are not sensitive to a factor of 2 variation in scaled streamwise pressure gradient over this range. Also, the 3 flight simulations shown thus far have had very different momentum thickness Reynolds numbers values varying from 62.4 to 136 . They, 
however, produced very similar heating bump factors, which shows an momentum thickness Reynolds number independence, over this range in $\mathrm{Re}_{\theta}$, for scaled cavities.

\section{Off-Centerline Flight Cavity}

Up to this point in the paper, only centerline cavity simulations aligned with the vehicle axis have been presented. For the flight simulations, this allows for the minimization of surface and streamline curvature and crossflow gradient effects on the heating bump factor scaling. A flight simulation was performed, however, off the windside centerline of the vehicle in an attempt to obtain some information on these effects. The cavity was located at a non-dimensional axial location $(\mathrm{X} / \mathrm{L})$ of 0.264 and was about 76 inches off the centerline at a $\mathrm{Y} / \mathrm{L}=0.06$. The cavity was positioned to be inline with the local velocity vector, causing the cavity to be angled at 21 degrees from the axial direction. The simulation was performed for CFD trajectory point 6 on the Orbiter STS-107 re-entry trajectory, give in table 3. This flight condition corresponds is 921 seconds from entry interface (EI). The edge properties are given in tables 1 . These values were an exact match of the wind tunnel values. The cavity dimensions are shown in table 2. The grid for the simulation is shown in figure 26 with a magnified view highlighting the grid inside the cavity shown in figure 27. The grid had 1.5 million cells with 502,000 cells inside the cavity. Grid refinements and iterative grid convergence checks were performed to ensure the grid was sufficiently refined and the solution met the convergence criteria. In addition a full Navier-Stokes simulation was also performed to ensure that the thin-layer assumption was valid. Figure 28 shows the heating bump factor contours about the cavity. The cavity heating indicates asymmetric behavior, with larger heating on the inboard downstream corner and less heating in the outboard downstream corner. Comparing these contours to those in figure 15 for the baseline centerline simulation, the peak heating levels are larger for the off-centerline cavity case. Figure 29 shows the streamlines about the cavity. While the cavity is inline with the incoming streamlines, due to the streamline curvature the downstream portions of the cavity are not inline with the velocity vectors, so one would not expect symmetric heating. The streamlines just inboard of the cavity are curved slightly outboard, into the cavity, near the back edge of the cavity. They impinge on the downstream face of the cavity producing increased heating on the back face. They also cause an increased heating on the downstream inboard portion of the cavity floor as the streamlines off the downstream face are redirected to the cavity floor. Figure 30 shows the cavity centerline and peak heating cuts for this cavity with the other flight cavities and the wind tunnel cavity simulations. While the centerline cut for the off-centerline cavity shows heating levels similar to the other flight cavities, the peak heating cut of the off-centerline cavity has much higher heating bump factor levels than the other flight cavities.

\section{Boundary Layer Profile Effects}

The flight simulations were observed to have much higher heating bump factors than the wind tunnel simulations. Figure 30, mentioned above, shows the streamwise cuts through the wind tunnel and flight cavities centerline and peak heating locations. It is clear that while the heating patterns are similar between the wind tunnel and flight simulations for all but the off-centerline flight cavity, the magnitude of the heating bump factors for flight are significantly larger than the wind tunnel values. The larger heating bump factors observed in flight is due to the the increased driving potential of the heating in the flight case. The increased driving potential refers to the increase in the relative amounts of energy the cavity sees for the flight simulations over the wind tunnel simulations. This increased driving potential is due to the fact that the rate of increase in total enthalpy increases with increasing height into the boundary layer is larger for the flight cases than for the wind tunnel case. This is shown in figure 31 which shows a scaled profile of the ratio of total enthalpy minus the wall enthalpy to the edge total enthalpy minus the wall enthalpy. The scaled height above the OML, Y/ $\delta$, is the ratio of the height above the OML to the boundary layer thickness. The total enthalpy profile was taken at a point just upstream of the cavity for both the wind tunnel and flight simulations. The enthalpy ratio for the flight simulation is rising at a rate of 2.5 times faster than the wind tunnel ratio as you increase the scaled height above the surface. This creates a larger driving function for the conduction of heat into the lower shear layers above the cavity. Also shown in figure 31 is the enthalpy ratio value and vertical location of the streamline which further downstream hits the stagnation point on the downstream wall of the cavity. The stagnation streamline has an enthalpy ratio value for the flight simulation which is $76 \%$ larger that the corresponding wind tunnel value. This is true even though the stagnation streamline originates from higher within the boundary layer, in a scaled sense, for the wind tunnel simulation. On a related note, figure 32 shows the Mach profiles just upstream of the cavity for the two simulations. The Mach number of the stagnation streamline for the flight simulation is 2.3 times larger than the wind tunnel simulation value. Therefore, there is more compressibility present in the flight cavity than the wind tunnel cavity. These profiles indicate that, for both the scaled enthalpy ratio and Mach number, even though we are matching the edge properties for the flight and wind tunnel simulations, we are not matching the enthalpy ratio and Mach number values that actually enter the cavity. 
Some the enthalpy in the lower levels of the boundary layer for the flight simulation is due to the energy tied up in the atom species present in this region. Figure 33 shows the contours of the mass fraction of molecular oxygen in a streamwise slice through the centerline of the cavity for the baseline flight simulation. While the boundary layer flow approaching the cavity has mass fraction levels of diatomic oxygen on the order of $1 \mathrm{e}-6$, at the flow impingement point on the downstream lip of the cavity the mass fraction rises 4 orders of magnitude. This is due to the recombination at the wall due to the catalytic action of the wall and the increased residence time of the flow near the stagnation point. The question became, however, how much did the recombination contribute to the heat transfer. The ratio of heat transfer due to diffusion to the total heat transfer at the wall is shown in figure 34 . At the reference point just upstream of the cavity, the diffusion account for $12 \%$ of the total heat transfer at the wall. At the downstream portion of the cavity floor the diffusive heating is $14 \%$ of the total heating while at the downstream lip the diffusive heating accounts for more than $40 \%$ of the total heating to the wall. While a significant fraction of energy is carried in the heat of formation of dissociated species for the flight cases, the impact of diffusion on relative heating rates can not be quantified.

This increased rate of enthalpy increase through the boundary layer also causes increased conduction of energy to the flow as it passes over the cavity, approaching the downstream lip. Figure 35 shows enthalpy ribbons for the streamlines which intersect the downstream face of the cavity near the stagnation point for the flight simulation. Figure 36 shows the analogous streamlines for the wind tunnel simulation. The contours on the volume ribbons are the total enthalpy along the streamline normalized by the wall enthalpy just upstream of the cavity. For the flight cases the enthalpy of the streamlines increases by a factor of 2.5 while for the wind tunnel simulation, the streamlines only increase in enthalpy about 55\%. This conduction is due to the larger enthalpy flow higher in the boundary layer conducting heat downward through the shear layers due to the absence of the cold wall driving function. For flight, the difference between the wall and edge total enthalpy was much larger than for the wind tunnel simulation, thereby creating a larger driving function with the wall present. When this cold wall presence is removed, conduction occurs more rapidly than for the wind tunnel simulation, with the large enthalpy levels within the flight boundary layer driving the conduction.

\section{Conclusions}

Wind tunnel and flight simulations were performed for open cavities at a variety of conditions. The wind tunnel and flight results were shown to be iteratively converged $\left(\Delta \mathrm{q}_{\mathrm{w}}<0.8 \%\right.$ over 1000 iterations) and grid converged. There appeared to be no difference between the wind tunnel simulations which were performed on a Cartesianaligned H-grid and a morphed O-grid, indicating grid topology independent solutions. For both the wind tunnel and flight simulations, the thin-layer and full Navier-Stokes simulations of the governing equations produced nearly identical results, indicating that the thin-layer formulation of the governing equations was sufficient to capture the flow physics.

The Orbiter windside centerline cavity flight simulations, performed at different locations and at different reentry trajectory points, showed similar results. The heating bump factors about the scaled cavities were not sensitive to variations in momentum thickness Reynolds number and streamwise scaled pressure gradients over the range considered. For the off-centerline windside cavity, an asymmetrical heating pattern was observed inside the cavity with larger peak heating values than were observed for the centerline cavity simulations. This was due to the streamline curvature in the vicinity of the cavity causing more focused impingement.

The wind tunnel and Orbiter windside centerline cavity flight simulations shows similar heating patterns in the cavity with increased heating observed at the downstream portion of the cavity floor and at the upper portion of the downstream face of the cavity, spilling onto the downstream OML. While the heating patterns were similar between the wind tunnel and flight simulation, the magnitudes of the heating bump factors were not. The peak bump factors values on the cavity floor for flight simulation were $67 \%$ larger than the peak wind tunnel simulation value. On the downstream face of the cavity the flight simulation values were $60 \%$ larger than the wind tunnel simulation values. On the OML downstream of the cavity, the flight values are about $20 \%$ larger than the wind tunnel values.

The increased levels of heating bump factor for the flight cases were due to a stronger total enthalpy gradient in the boundary layer feeding the cavity. A significant fraction of energy is carried in the heat of formation of dissociated species for the flight cases; however, the impact of diffusion on relative heating rates can not be quantified. 


\section{References}

${ }^{1}$ Pulsonetti, M. V., Thompson, R. A, and Alter, S. J., "Prediction of STS-107 Hypervelocity Flow Fields about the Shuttle Orbiter with Various Wing Leading Edge Damage,” JANNAF Conference, Colorado Springs, Colorado, 2003

${ }^{2}$ Pulsonetti, M. V., and Thompson, R. A, "LAURA Aerothermodynamic Computations for Space Shuttle Columbia STS-107 Baseline and Damage," AIAA 35 ${ }^{\text {th }}$ Fluid Dynamics Conference, Portland, Oregon, 2004

${ }^{3}$ Reuther, J., Thompson, R., Pulsonetti, M. V., Campbell, C., "External Computational Aerothermodynamics Analysis for the STS-107 Accident Investigation,” AIAA 42 ${ }^{\text {nd }}$ Aerospace Sciences Meeting and Exhibit, Reno, Nevada, 2004

4,'Columbia Accident Investigation Report”, Report Volume I, August 2003

${ }^{5}$ Everhardt, J. L., Bey, K. S., Wood, W. A., and Pulsonetti, M. V., "Experimental and Computational Investigation of Cavity Flow Simulating Debris Damage to Thermal Protection System Tiles,” JANNAF Conference, Colorado Springs, Colorado, 2003

${ }^{6}$ Gnoffo, P. A., Gupta, R. N., and Shinn, J., "Conservation Equations and Physical Models for Hypersonic Air Flows in Thermal and Chemical Nonequilibrium,” NASA TP 2867, 1989

${ }^{7}$ Gnoffo, P. A., “An Upwind-Biased, Point-Implicit Relaxation Algorithm for Viscous, Compressible Perfect-Gas Flows,” NASA TP 2953, 1990

${ }^{8}$ Weilmuenster, K.J. and Gnoffo, P.A., “Solution Strategy for Three-Dimensional Configurations at Hypersonic Speeds,” Journal of Spacecraft an Rockets, Vol. 30, No. 4, July-August 1993, pp. 385-394

${ }^{9}$ Gnoffo, P. A., and Weilmuenster, J.J., "Multiblock Analysis for Shutlle Orbiter Re-Entry Heating from Mach 24 to Mach 12,” AIAA Paper 93-2813, July 1993

${ }^{10}$ Roe, P. L., “Approximate Riemann Solvers, Parameter Vectors and Difference Schemes,” Journal of Computational Physics, Vol. 43, No. 2, 1981, pp. 357-372.

${ }^{11}$ Harten, A., "High Resolution Schemes for Hyperbolic Conservation Laws," Journal of Computational Physics, Vol. 49, No. 3, 1983, pp. 357-393.

${ }^{12}$ Yee, H. C., “On Symmetric and Upwind TVD Schemes,” NASA TM 88325, 1990.

${ }^{13}$ Fay, J.A., and Riddell, F.R., “Theory of Stagnation Point Heat Transfer in Dissociated Air,” Journal of Aeronautical Sciences, Vol. 25, No. 2, pp. 73-85, Feb. 1958.

${ }^{14}$ Stewart, D. A., "Surface Catalysis and Characterization of Proposed Candidate TPS for Access-to-Space Vehicles," NASA TM 112206, 1997

${ }^{15}$ Charwat, A.F., Roos, J.N., Dewey, F.C. Jr., and Hitz, J.A., “An Investigation of Separated Flows - Part I: The Pressure Field”, Journal of the Aerospace Sciences, June 1961

${ }^{16}$ Charwat, A.F., Dewey, F.C. Jr., Roos, J.N., and Hitz, J.A., “An Investigation of Separated Flows - Part II: Flow in the Cavity and Heat Transfer”, Journal of the Aerospace Sciences, July 1961

${ }^{17}$ Gnoffo, P. A., “Grid Morphing Users Manual - Version 1.0”, NASA Langley Internal Publication, 2005

${ }^{18}$ Fletcher, L.S., Briggs, D.G., and Page, R.H., “A Review of Heat Transfer in Separated and Reattached Flows”, AIAA-70767,1970

${ }^{19}$ Nestler, D.E., “The Effects of Surface Discontinuities on Convective Heat Transfer in Hypersonic Flow”, AIAA-85-0971, AIAA $20^{\text {th }}$ Thermophysics Conference, Williamsburg, Virginia, June 1985

${ }^{20}$ Hunt, L.R., “Aerodynamic Heating in Large Cavities in an Array of RSI Tiles”, NASA TN D-8400, May 1977

${ }^{21}$ Nestler, D. E., Saydah, A. R., and Auxer, W. L., "Heat Transfer to Steps and Cavities in Hypersonic Turbulent Flow”, AIAA Paper No. 68-673, June 1968

${ }^{22}$ Hahn, M., "Experimental Investigation of Separated Flow over a Cavity at Hypersonic Speed,” AIAA Journal, Vol. 7, No. 7, June 1969, pp. 1092-1098

\section{Table 1: Edge Properties of Wind Tunnel and Flight Cavities}




\begin{tabular}{|c|c|c|c|c|c|}
\hline Edge Property & $\begin{array}{c}\text { Wind Tunnel } \\
\text { Run 247 }\end{array}$ & $\begin{array}{c}\text { Flight } \\
\text { CL Cavity } \\
\text { X/L=0.15 } \\
\text { CFD pt. 1 }\end{array}$ & $\begin{array}{c}\text { Flight } \\
\text { CL Cavity } \\
\text { X/L=0.15 } \\
\text { CFD pt. 2 }\end{array}$ & $\begin{array}{c}\text { Flight } \\
\text { CL Cavity } \\
\text { X/L=0.05 } \\
\text { CFD pt. 1 }\end{array}$ & $\begin{array}{c}\text { Flight } \\
\text { Off-CL Cavity } \\
\text { X/L=0.26 } \\
\text { Y/L=0.06 } \\
\text { CFD pt 6 }\end{array}$ \\
\hline $\mathrm{Re}_{\theta}$ & 260 & 116 & 136 & 62.4 & 260 \\
\hline$M_{\mathrm{e}}$ & 2.25 & 2.18 & 2.18 & 1.49 & 2.25 \\
\hline$\delta$ (in) & 0.131 & 2.35 & 2.15 & 1.50 & 1.21 \\
\hline
\end{tabular}

Table 2: Wind Tunnel and Flight Cavity Dimensions

\begin{tabular}{|c|c|c|c|c|c|}
\hline Dimensions & $\begin{array}{c}\text { Wind Tunnel } \\
\text { Run 247 }\end{array}$ & $\begin{array}{c}\text { Flight } \\
\text { CL Cavity } \\
\text { X/L=0.15 } \\
\text { CFD pt. 1 }\end{array}$ & $\begin{array}{c}\text { Flight } \\
\text { CL Cavity } \\
\text { X/L=0.15 } \\
\text { CFD pt. 2 }\end{array}$ & $\begin{array}{c}\text { Flight } \\
\text { CL Cavity } \\
\text { X/L=0.05 } \\
\text { CFD pt. 1 }\end{array}$ & $\begin{array}{c}\text { Flight } \\
\text { Off-CL Cavity } \\
\text { X/L=0.26 } \\
\text { Y/L=0.06 } \\
\text { CFD pt 6 }\end{array}$ \\
\hline L (in) & 0.75 & 13.4 & 12.2 & 8.53 & 6.87 \\
\hline W (in) & 0.51 & 9.07 & 8.28 & 5.76 & 4.65 \\
\hline H (in) & 0.10 & 1.79 & 1.63 & 1.14 & 0.92 \\
\hline
\end{tabular}

Table 3: Flight STS-107 CFD Trajectory Points

\begin{tabular}{|c|c|c|c|}
\hline $\begin{array}{c}\text { Freestream } \\
\text { Property }\end{array}$ & CFD pt 1 & CFD pt 2 & CFD pt 6 \\
\hline $\begin{array}{c}\text { Alt } \\
(\mathrm{km})\end{array}$ & 74.1 & 72.1 & 61.2 \\
\hline Mach \# & 24.8 & 24.2 & 17.9 \\
\hline Angle of Attack (deg) & 40.2 & 40.0 & 39.0 \\
\hline
\end{tabular}




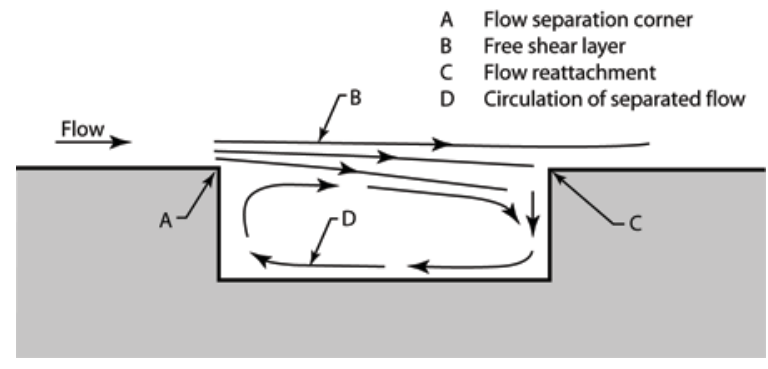

Figure 1. Schematic of Two-Dimensional Open Cavity Behavior (Modified from ref. 19 and 20)

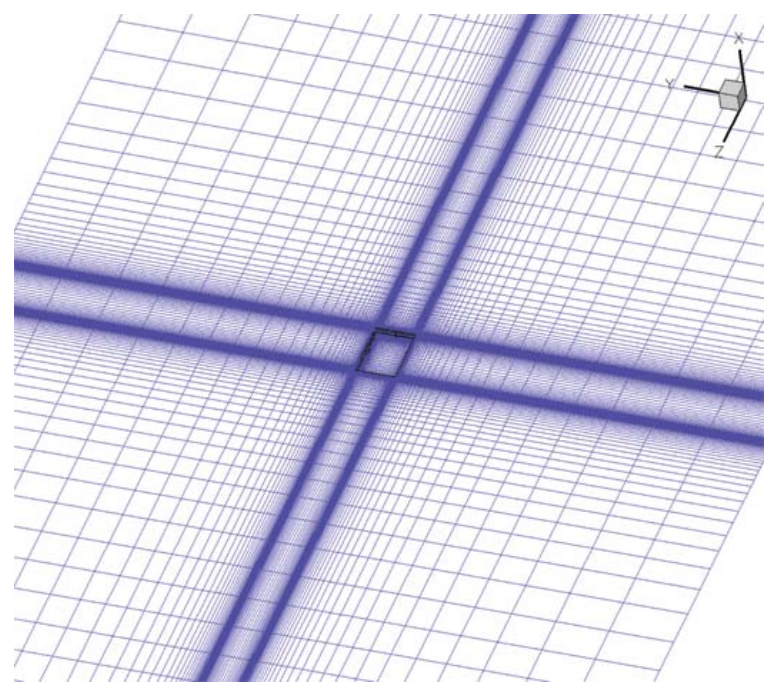

Figure 2. Cartesian-Aligned Cavity Grid for Wind Tunnel Simulation

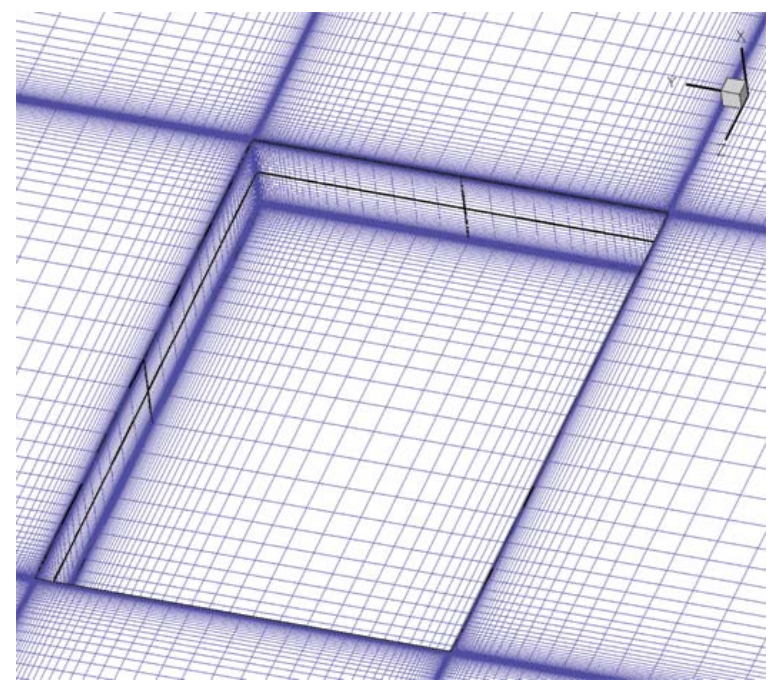

Figure 3. Magnified View of Cartesian-Aligned Cavity Grid for Wind Tunnel Simulation

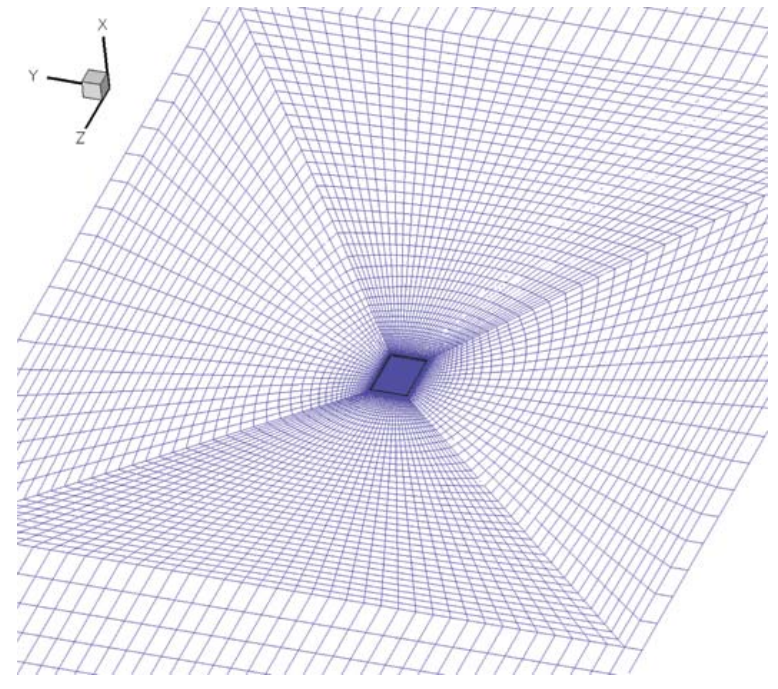

Figure 4. Morphed Cavity Grid for Wind Tunnel Simulation

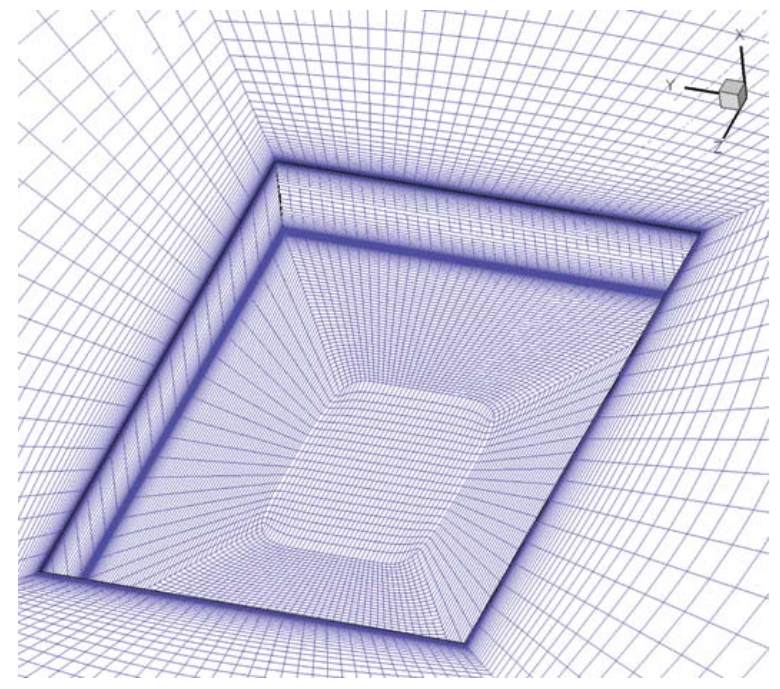

Figure 5. Magnified View of Morphed Cavity Grid for Wind Tunnel Simulation 


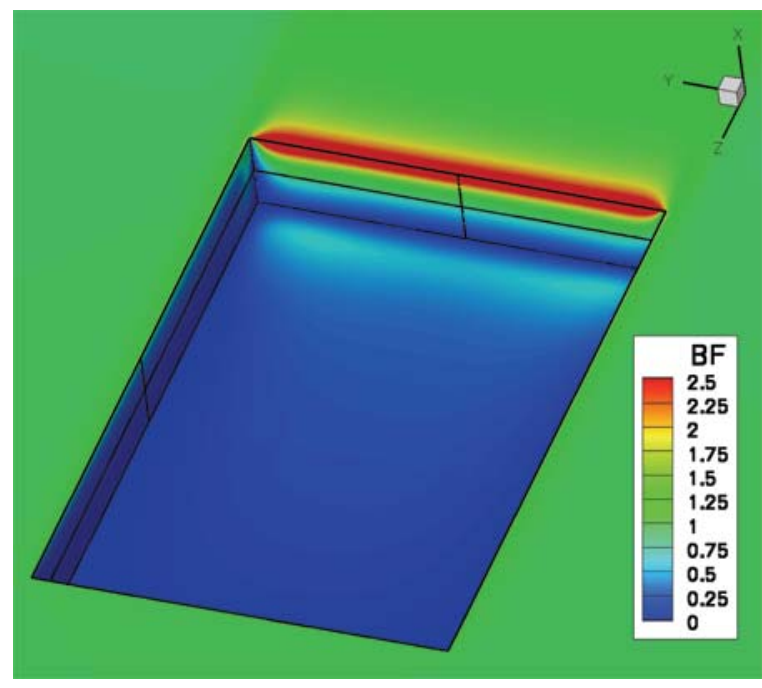

Figure 6. Heating Bump Factors for CartesianAligned Cavity Grid Wind Tunnel Cavity Simulation

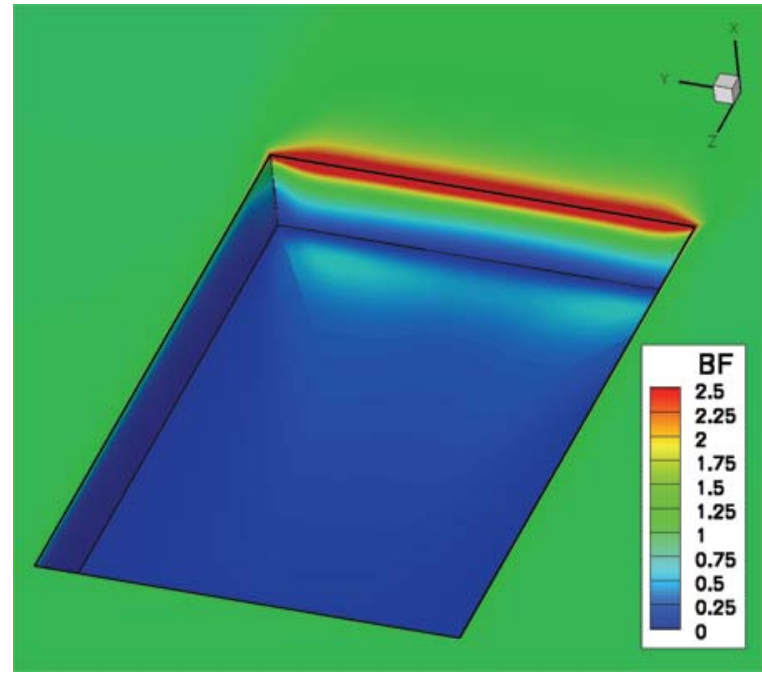

Figure 7. Heating Bump Factors for Morphed Cavity Grid Wind Tunnel Cavity Simulation

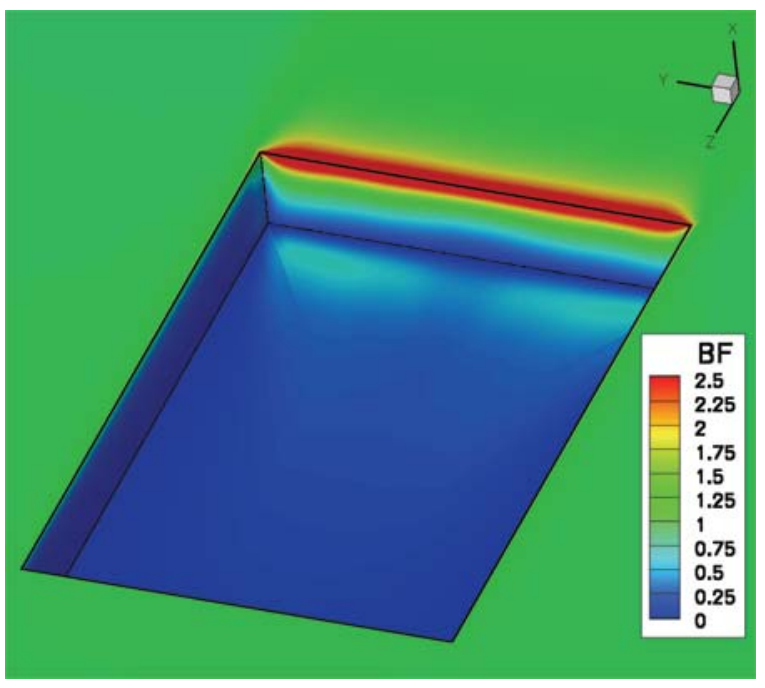

Figure 8. Heating Bump Factors for Morphed Fine Grid Simulation

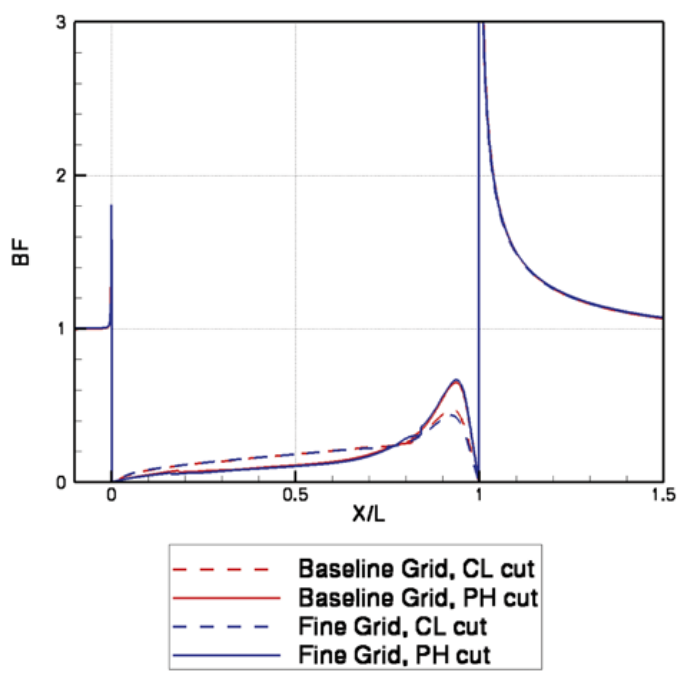

Figure 9. Centerline and Peak Heating Cuts of Morphed Baseline and Fine Grid Wind Tunnel Simulations 


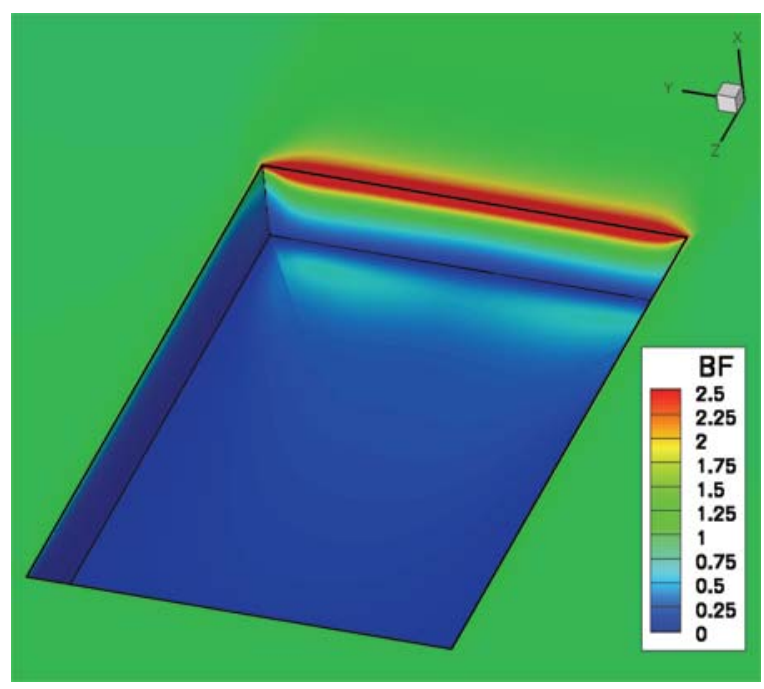

Figure 10 Heating Bump Factors for Full NavierStokes Wind Tunnel Simulation on Morphed Grid

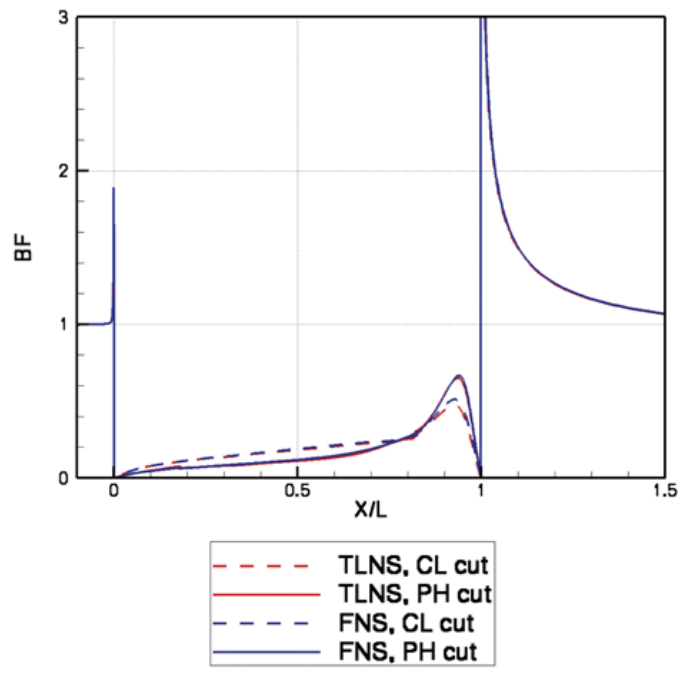

Figure 11. Centerline and Peak Heating Cuts of Thin-Layer and Full Navier-Stokes Wind Tunnel Simulations on Morphed Grid

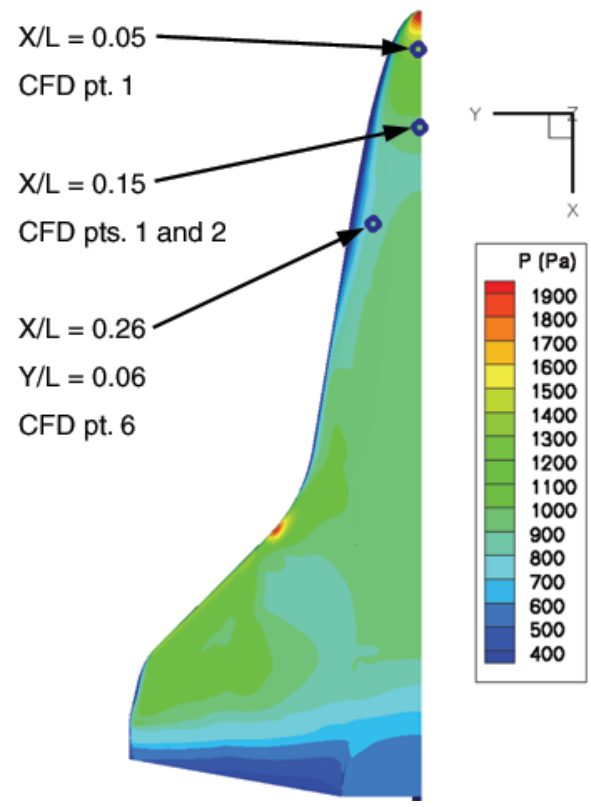

Figure 12. Cavity Locations for the Flight Simulations

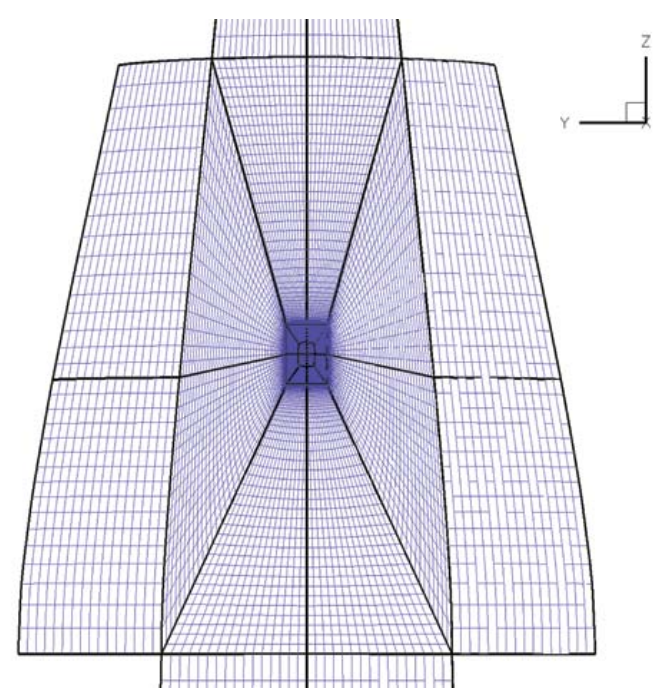

Figure 13. Morphed Cavity Grid for Flight Simulations at $\mathrm{X} / \mathrm{L}=0.15$ 


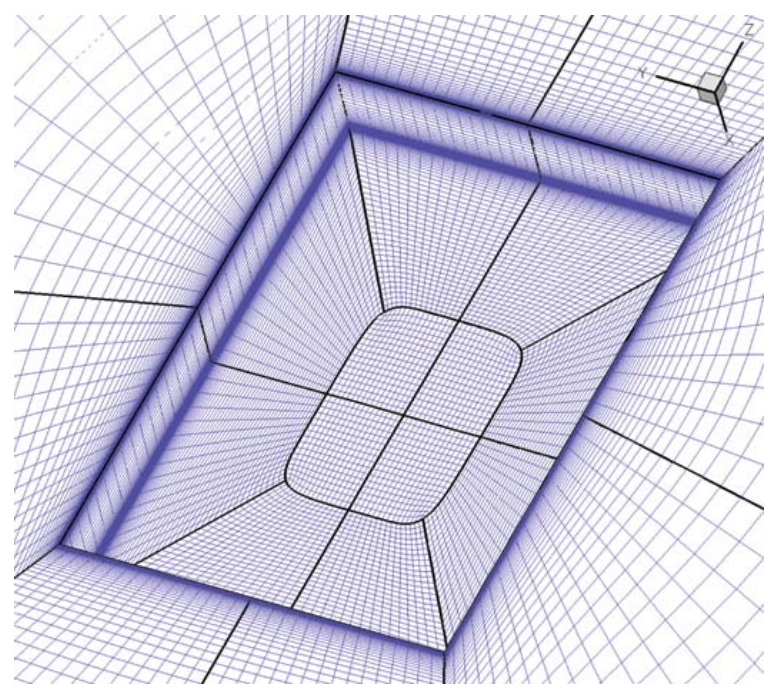

Figure 14. Magnified View of Morphed Cavity Grid for Flight Simulations at X/L=0.15

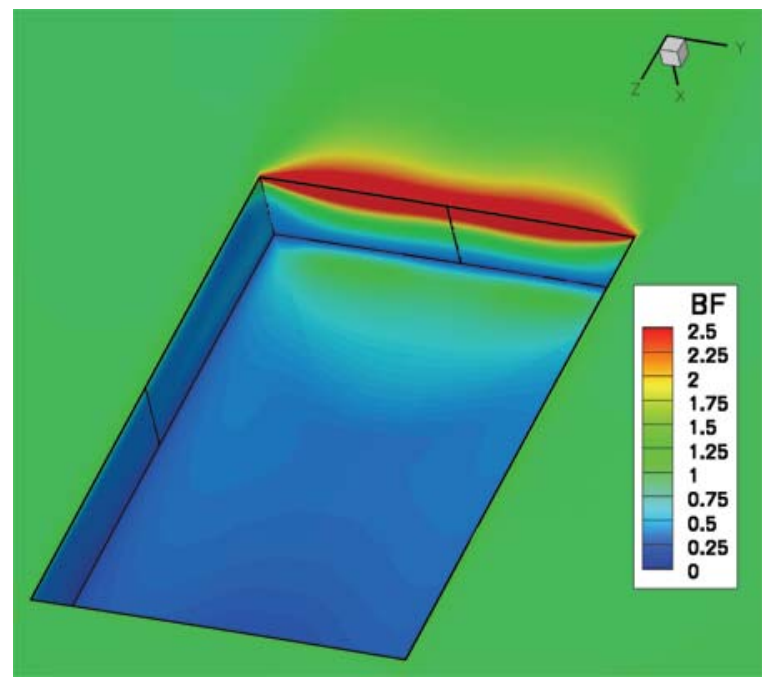

Figure 15. Heating Bump Factor Contours for Flight Simulation at $X / L=0.15$ for $C F D$ point 1

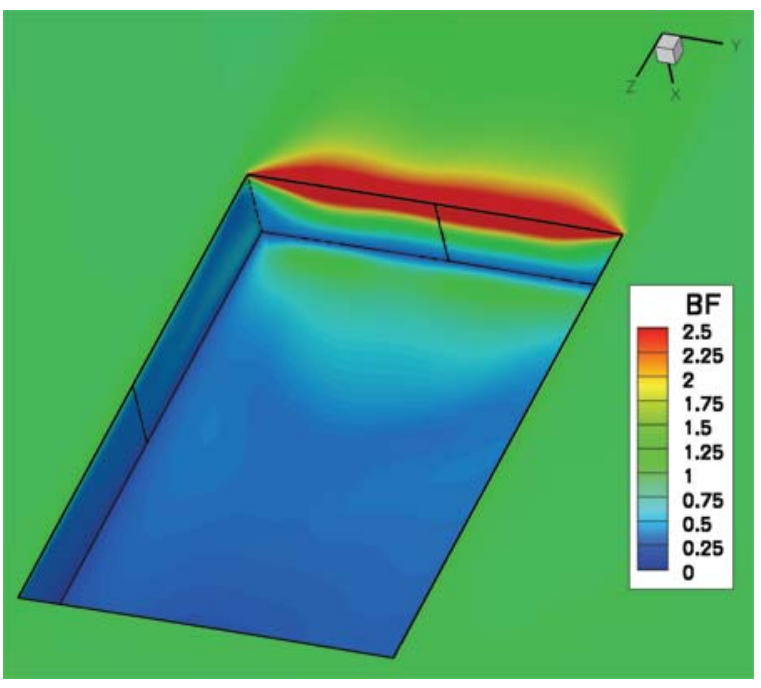

Figure 16. Heating Bump Factors for Flight Simulation at $X / L=0.15$ for CFD point 1 on a Fine Grid

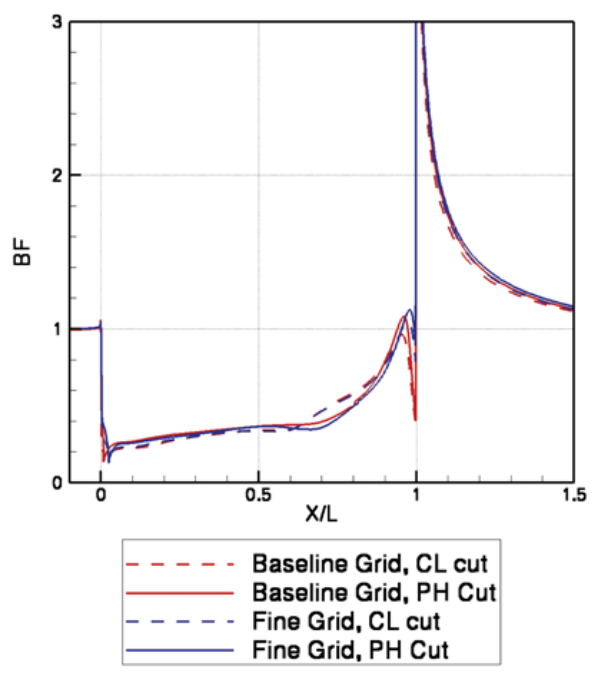

Figure 17. Centerline and Peak Heating Cuts of Flight Simulation at $\mathrm{X} / \mathrm{L}=0.15$ for $\mathrm{CFD}$ point 1 on the Baseline and Fine Grid 


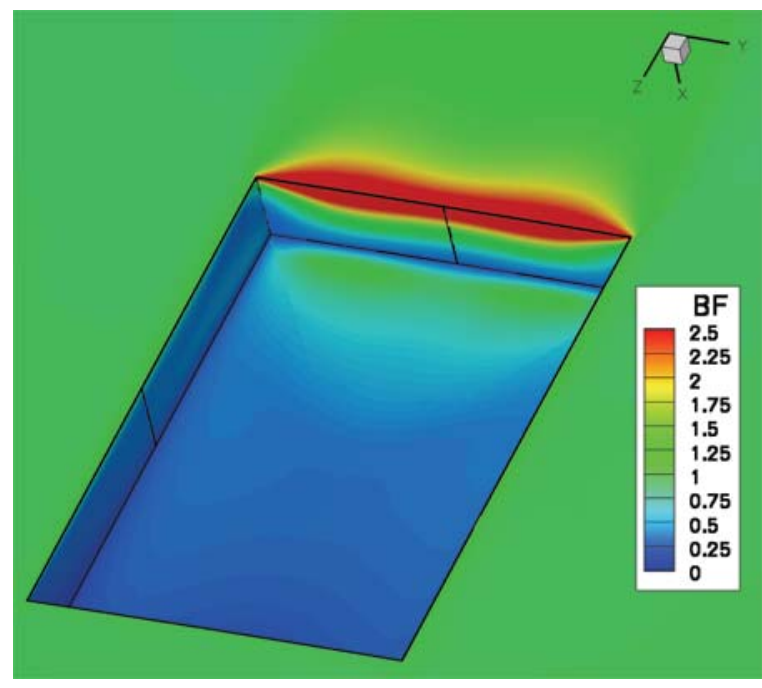

Figure 18. Heating Bump Factors for Full Navier-Stokes Flight Simulation at $X / L=0.15$ for CFD point 1

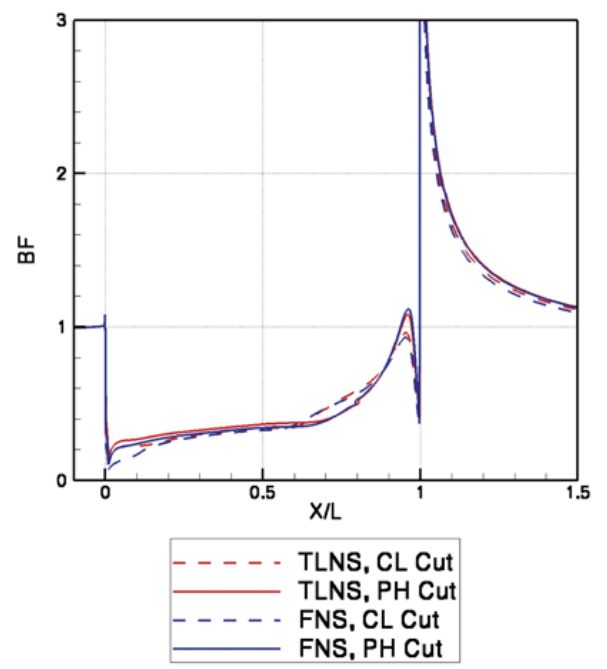

Figure 19. Centerline and Peak Heating Cuts of Thin-Layer and Full Navier-Stokes Flight Simulations at $X / L=0.15$ for CFD point 1

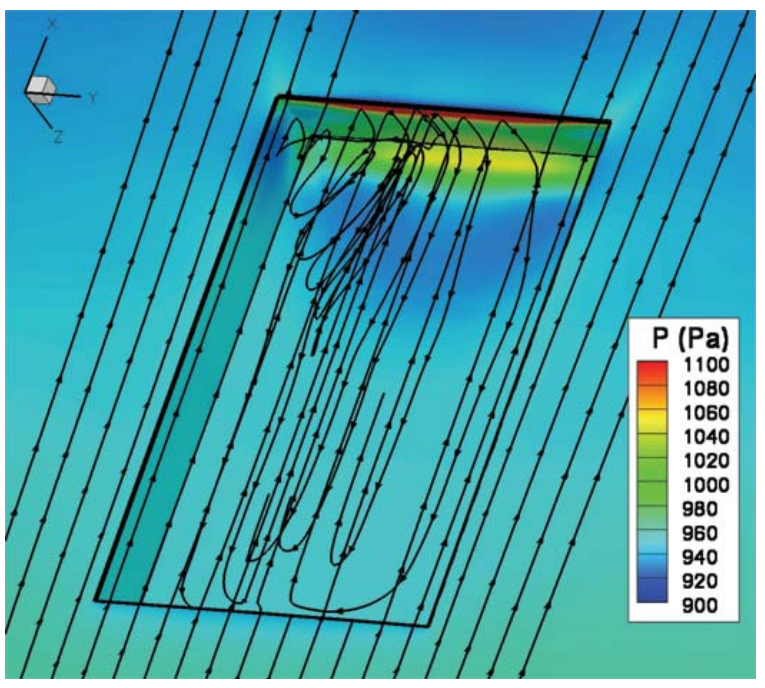

Figure 20. Pressure Contours and Volume Streamlines for Flight Simulation at $X / L=0.15$ for CFD point 1

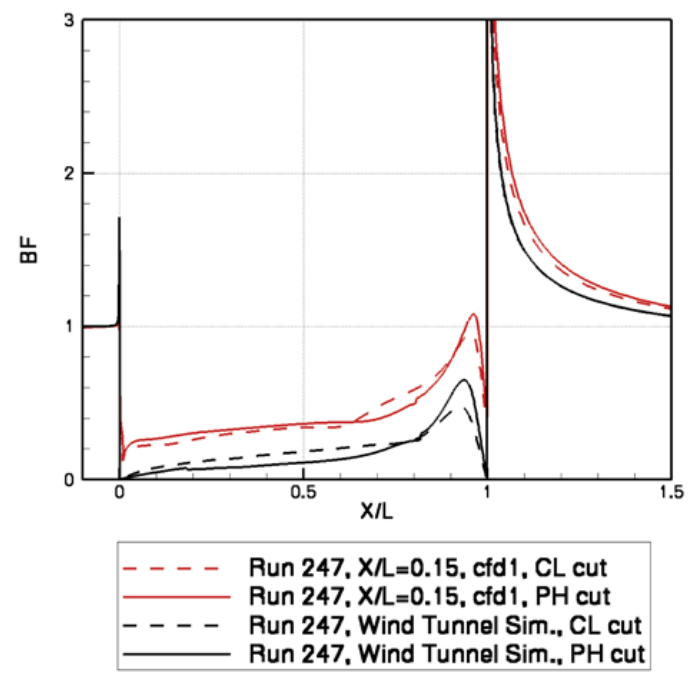

Figure 21. Centerline and Peak Heating Cuts of the Baseline Wind Tunnel and Flight Simulation 


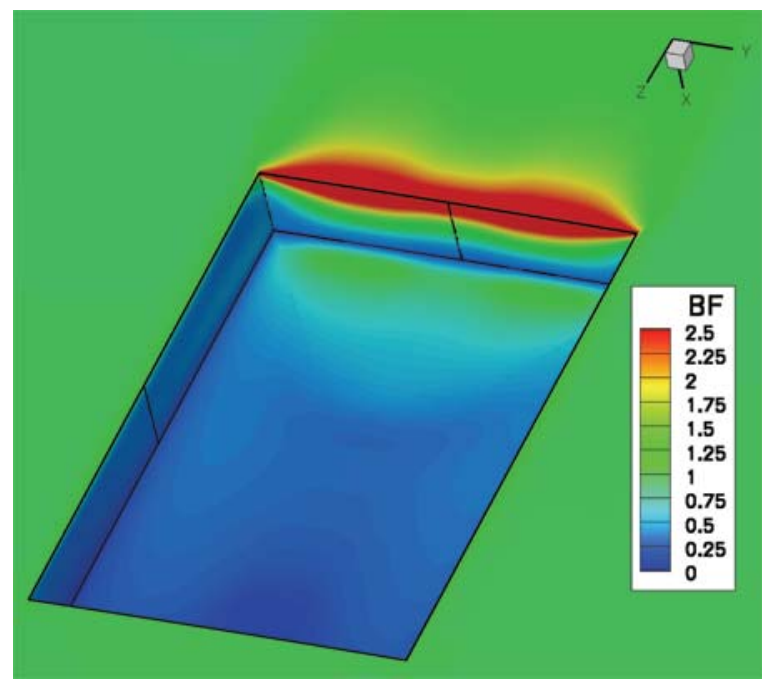

Figure 22. Heating Bump Factor Contours for Flight Simulation at $X / L=0.15$ for CFD point 2
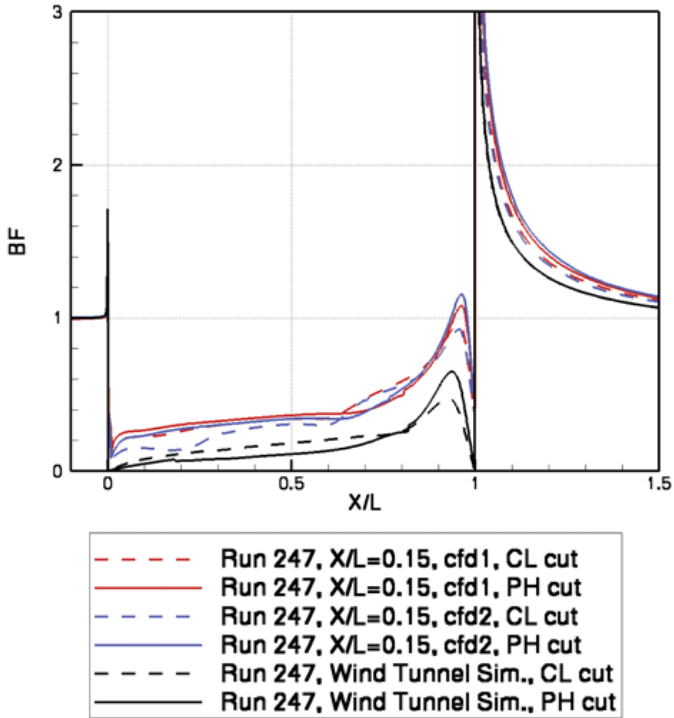

Figure 23. Centerline and Peak Heating Cuts of Wind Tunnel and two Flight Simulations at CFD points 1 and 2

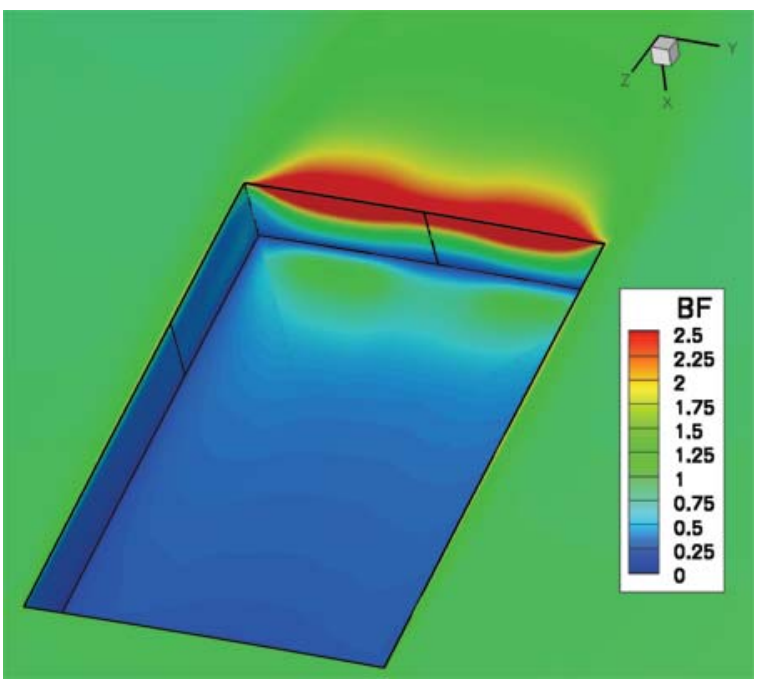

Figure 24. Heating Bump Factor Contours for Flight Simulation at $X / L=0.05$ for CFD point 1

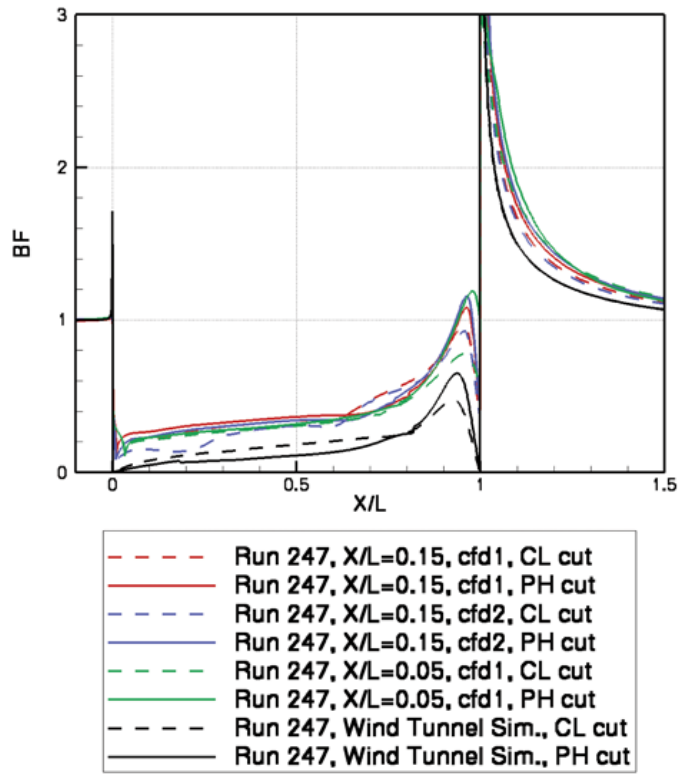

Figure 25. Centerline and Peak Heating Cuts of Wind Tunnel and three Flight Simulations at CFD points 1 and 2 


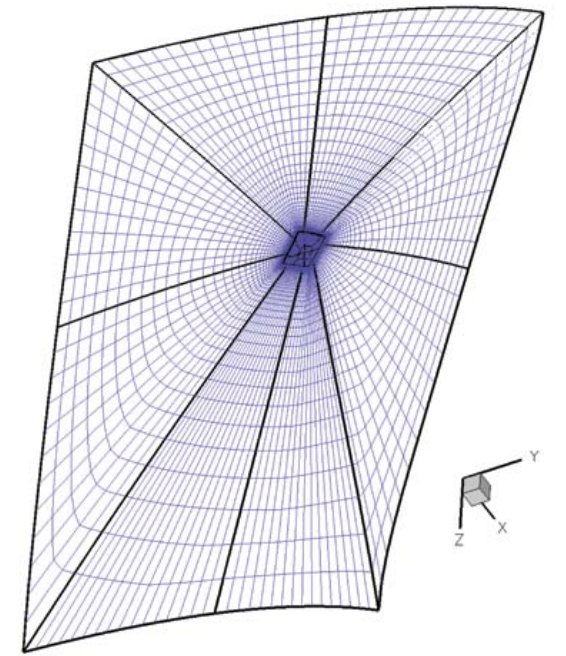

Figure 26. Morphed Cavity Grid for OffCenterline Flight Simulation

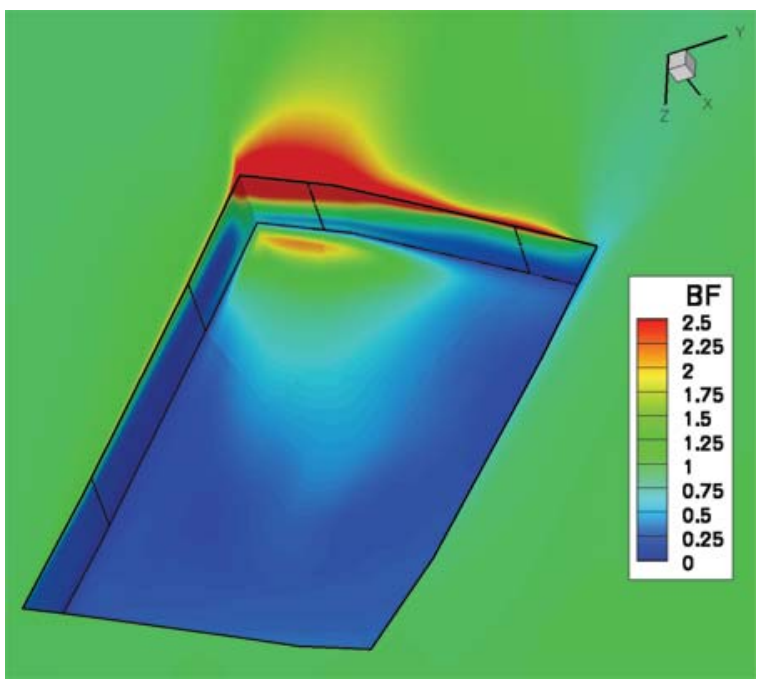

Figure 28. Heating Bump Factor Contours for Off-Centerline Flight Simulation at CFD point 6

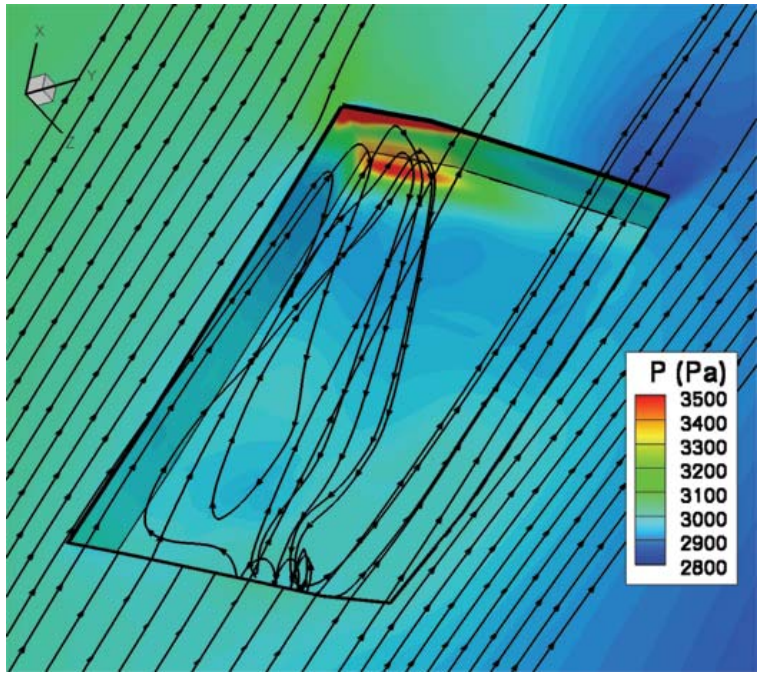

Figure 29. Pressure Contours and Volume Streamlines for Off-Centerline Flight Simulation at CFD point 6 

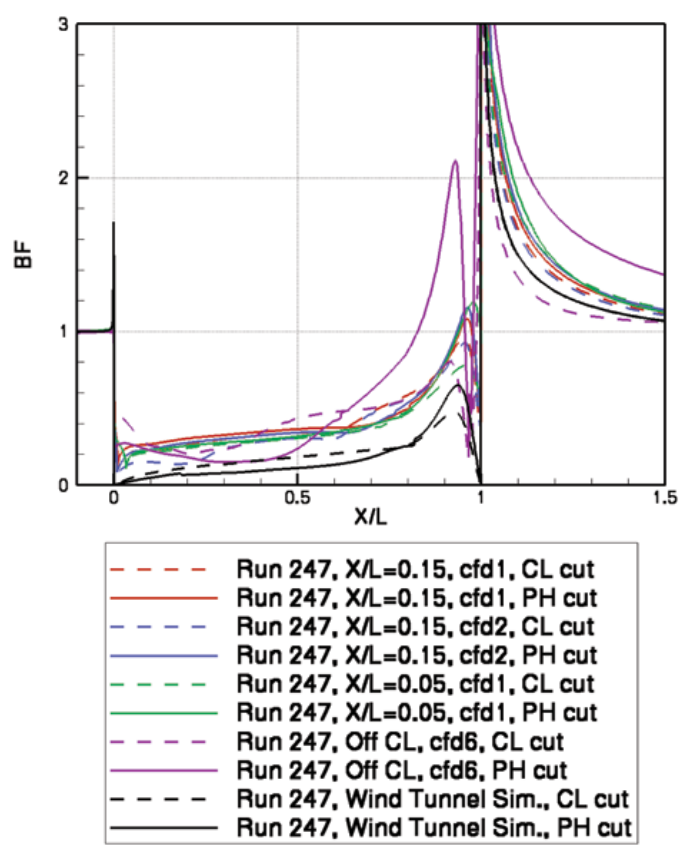

Figure 30. Centerline and Peak Heating Cuts of Flight and Wind Tunnel Simulations

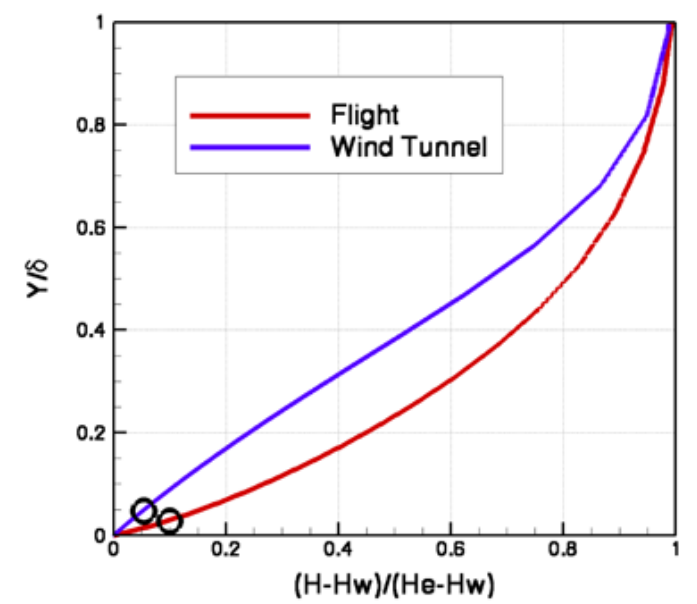

Figure 31. Enthalpy Ratio Profiles for the Baseline Wind Tunnel and Flight Simulations

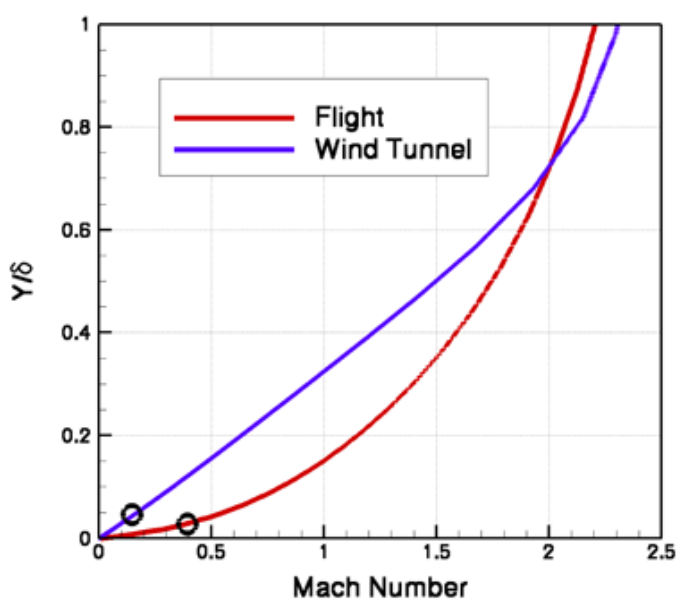

Figure 32. Mach Number Profiles for the Baseline Wind Tunnel and Flight Simulations

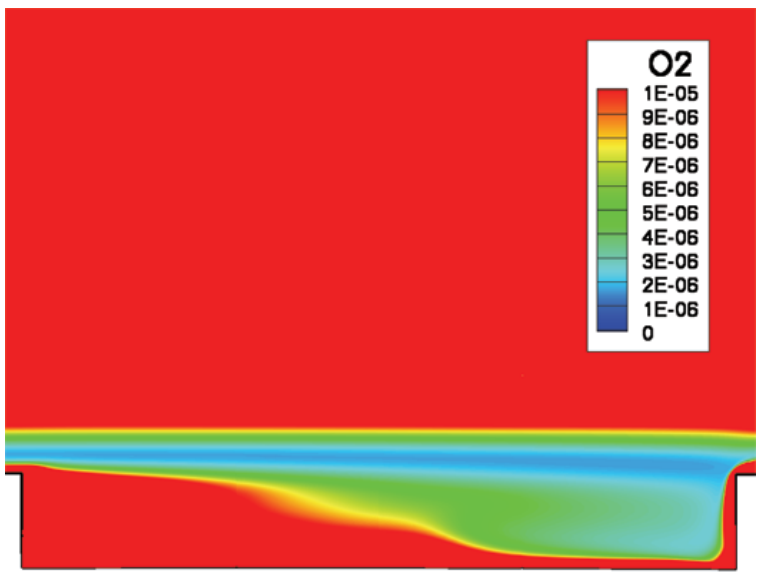

Figure 33. Mass Fraction of Diatomic Oxygen in Cavity for Flight Simulation at $X / L=0.15$ for CFD Point 1 


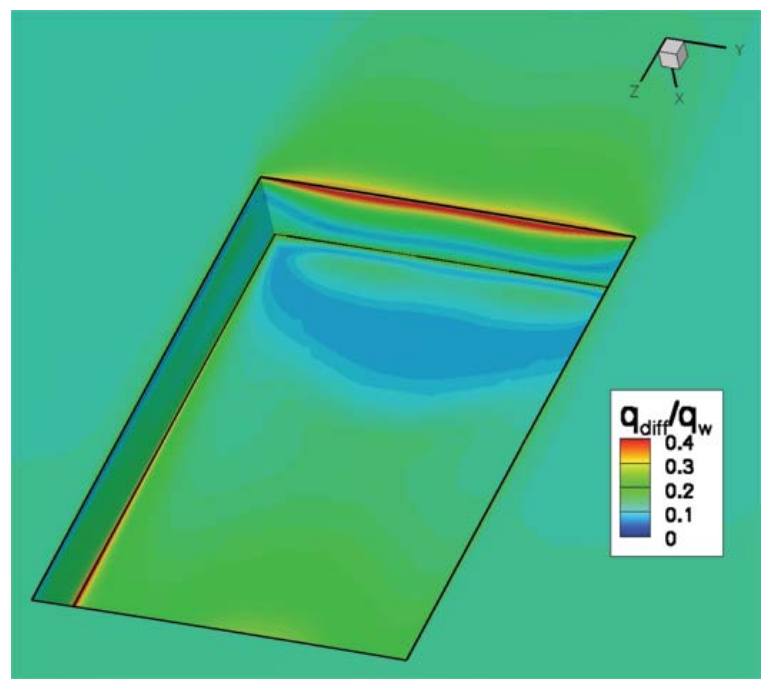

Figure 34. Ratio of Diffusion Component of Heating to Total Wall Heating for Flight Simulation at $\mathrm{X} / \mathrm{L}=0.15$ for CFD Point 1

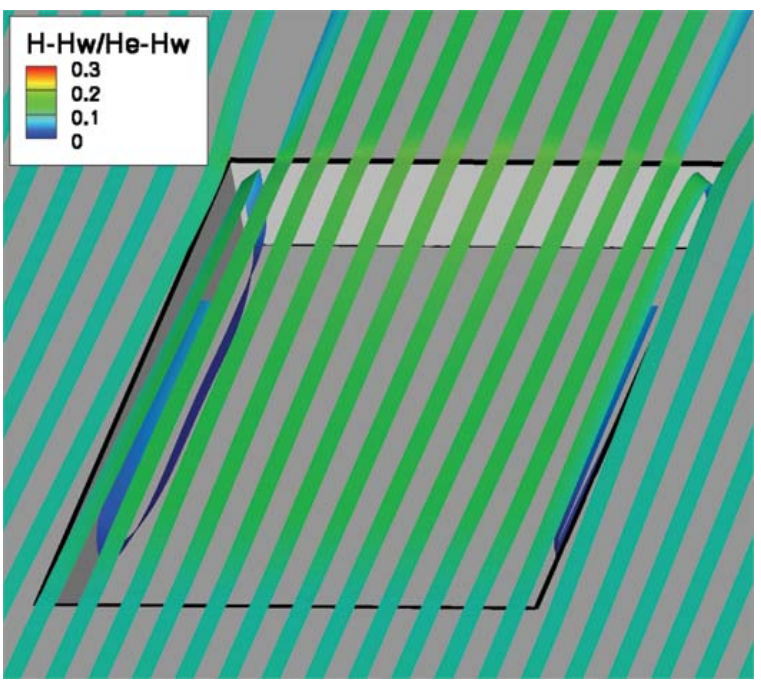

Figure 36. Enthalpy Volume Ribbons for the Wind Tunnel Simulation

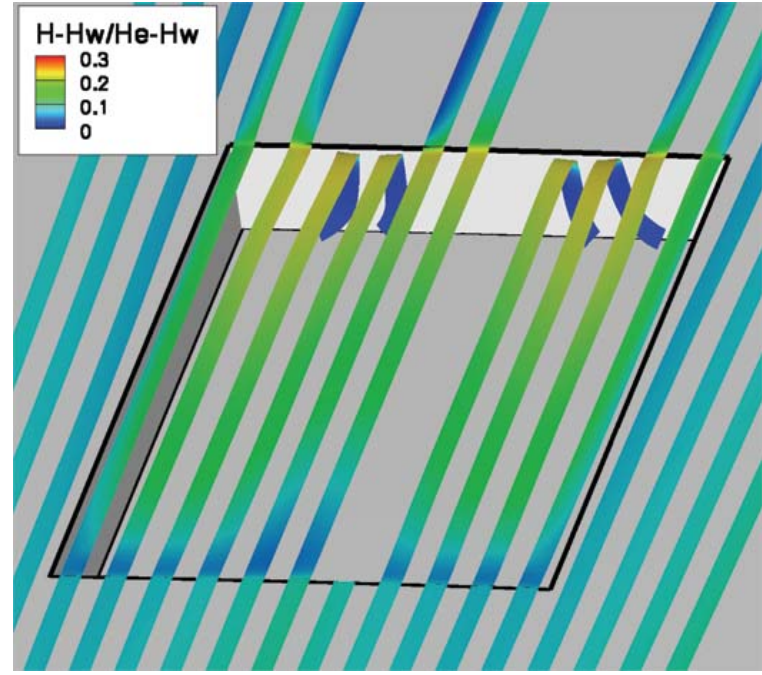

Figure 35. Enthalpy Volume Ribbons for the Flight Simulation at $\mathrm{X} / \mathrm{L}=0.15$ for CFD Point 1 